\title{
The role of port site local anesthetic injection in laparoendoscopic single site surgery: a prospective randomized study
}

\author{
Jong Wook Seo, MD', In Ok Lee, MD', Jung Cheol Kim, MD², Jae Eun Chung, MD' \\ Department of Obstetrics and Gynecology, ${ }^{1}$ National Health Insurance Service Ilsan Hospital, Goyang, ${ }^{2}$ Yonsei University, Severance Hospital, Seoul, \\ Korea
}

\section{Objective}

To investigate the role of port-site bupivacaine hydrochloride injection in laparoendoscopic single-site surgery (LESS) as a means of postoperative umbilical pain alleviation.

\section{Methods}

A total of 200 consecutive patients who underwent LESS from October 2018 to February 2019 were included in this randomized prospective case control study. The patients were alternatively assigned to either the study group $(0.25 \%$ $10-\mathrm{mL}$ bupivacaine hydrochloride injection at the 1.5-cm umbilical incision site after surgery) or the control group (no injection). All patients underwent surgery at the National Health Insurance Service Ilsan Hospital under the same operational setting by 3 board-certified gynecologists. Postoperative umbilical pain scores assessed using the visual analog scale were compared between the 2 groups as the primary outcome. Student's $t$-test, $\chi^{2}$ test, and a linear mixed model were used for the statistical analysis. A $P$-value of $<0.05$ was considered to be statistically significant.

\section{Results}

The patients' age, body mass index, and menopausal status; type of surgery performed; and need for additional trocar insertion exhibited a significant difference between the bupivacaine injection and non-injection groups. After adjusting for various confounding variables, the postoperative umbilical pain scores measured at postoperative 2-3 hours, 6-10 hours, 1 day, and 3 days did not exhibit a significant difference between the 2 groups.

\section{Conclusion}

Port-site bupivacaine injection in LESS did not show any additive effect in alleviation of postoperative umbilical pain.

Keywords: Laparoscopic surgery; Gynecologic surgical procedure; Postoperative pain; Bupivacaine hydrochloride

\section{Introduction}

Compared to open surgeries, laparoendoscopic gynecologic surgeries confer various benefits, such as shorter postoperative hospital stay, aesthetic superiority of the incision site, earlier return to daily activities, and decreased postoperative pain [1-4].

For alleviation of postoperative pain, techniques, such as preoperative and postoperative intraperitoneal infusions of local anesthetics and direct injection of local anesthetics at the incision site, have been used [5-9]. In laparoendoscopic single-site surgery (LESS) where only 1 trocar-site incision is created, port-site injection of local anesthetics, such as bupi-
Received: 2019.07.23. Revised: 2019.11.19. Accepted: 2019.12.18. Corresponding author: Jae Eun Chung, MD

Department of Obstetrics and Gynecology, National Health Insurance Service Ilsan Hospital, 100 Ilsan-ro, Ilsandong-gu, Goyang 10444, Korea

E-mail: obgy0278@nhimc.or.kr; jiupark@naver.com https://orcid.org/0000-0003-0146-6286

Articles published in Obstet Gynecol Sci are open-access, distributed under the terms of the Creative Commons Attribution Non-Commercial License (http://creativecommons. org/licenses/by-nc/3.0/) which permits unrestricted non-commercial use, distribution, and reproduction in any medium, provided the original work is properly cited.

Copyright (c) 2020 Korean Society of Obstetrics and Gynecology 


\section{Obstetrics \& Gynecology Science}

Vol. 63, No. 3, 2020

vacaine hydrochloride or lidocaine has been commonly performed for postoperative pain reduction, as it is a relatively safe and economically feasible procedure. Conflicting results have been reported on the effect of port-site local anesthesia in gynecologic laparoendoscopy; however, the reporting studies had a retrospective design, had the procedures performed by a single surgeon, or included relatively simple, benign, adnexal surgeries with an operation time of less than 1 hour [10-13].

In this institution, almost all the gynecologic surgeries were performed with LESS, except in cases necessitating open procedures, such as ovarian malignancy with metastatic lesions. We sought to analyze the role of trocar-site bupivacaine injection in postoperative umbilical incisional pain alleviation in LESS, including various procedures, such as hysterectomy, myomectomy, cystectomy, and robot-assisted LESS, in this prospective, randomized trial.

\section{Materials and methods}

\section{Study population}

A total of 223 gynecologic surgeries were performed by 3 board-certified gynecology surgeons from October 2018 to February 2019 at the Department of Obstetrics and Gynecology, National Health Insurance Service Ilsan Hospital, Korea.
The excluded cases are shown in Fig. 1. No patients had a history of bupivacaine allergy. Patient characteristics of age, parity, body mass index $\left(\mathrm{BMl}, \mathrm{kg} / \mathrm{m}^{2}\right)$, and menopausal status; associated comorbidities, including hypertension and diabetes mellitus; American Society of Anesthesiologist (ASA) score; history of abdominal surgery; and abdominal visual analog scale (VAS) pain score prior to surgery were assessed for group comparison. The procedures performed; final pathologic diagnosis; blood loss during surgery; hemoglobin changes after surgery; need for additional trocar usage; perioperative complications, including bowel injury and infection; operation time from skin incision to closure; need for packed red blood cell transfusion; need for postoperative rescue doses of analgesia other than the routinely applied intravenous patient-controlled anesthesia (IV-PCA); hospital stay after surgery in days; and time of flatus in hours were also assessed.

With the 23 exclusions, a total of 200 patients who signed the informed consent form were finally enrolled and alternatively assigned to either the bupivacaine injection or the noninjection group in a consecutive manner.

\section{Surgical procedure}

All surgeries were performed under general anesthesia with endotracheal intubation. Intraoperative analgesics, such as fentanyl, morphine, and pethidine, were administered in

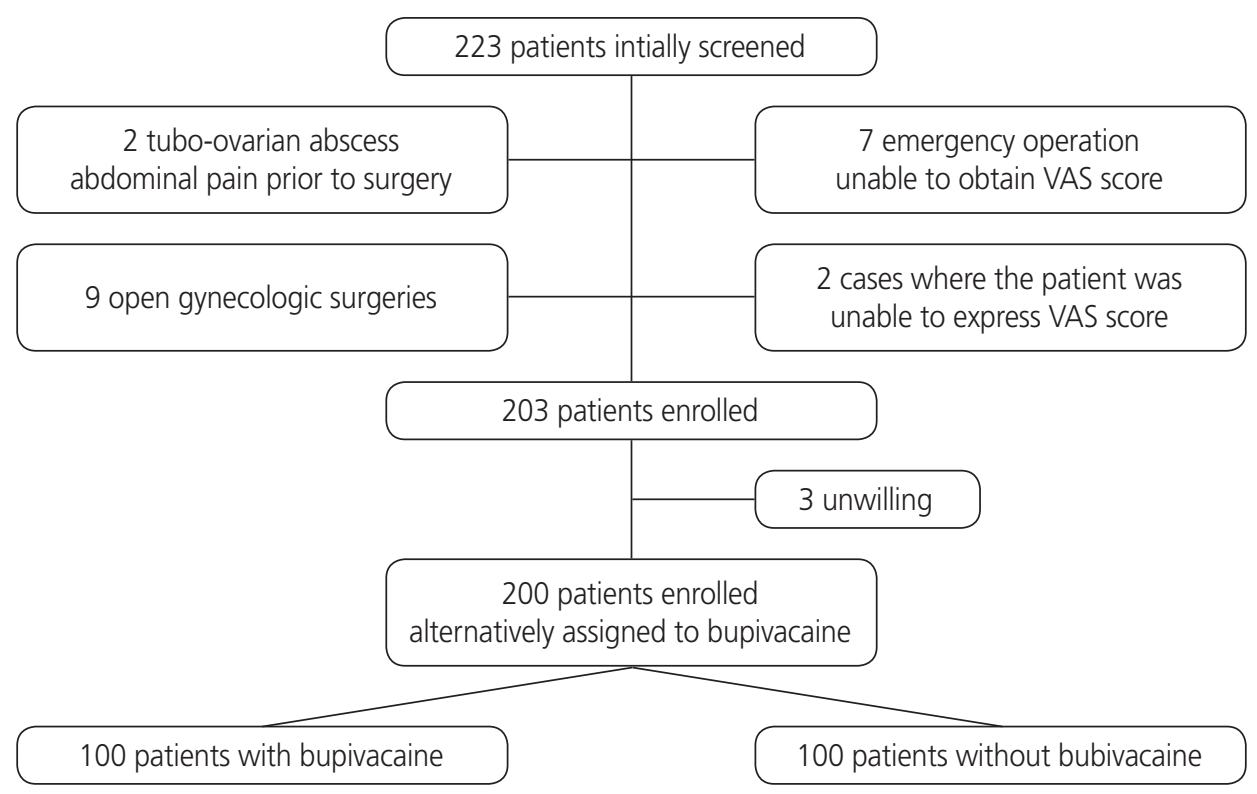

Fig. 1. Patient selection. VAS, visual analog scale. 


\section{Obstetrics \& Gynecology Science}

Jong Wook Seo, et al. Pain management in laparoscopic surgery

accordance with the standardized protocol held by the Department of Anesthesiology at the National Health Insurance Service Ilsan Hospital. With the patients in the low lithotomy position, the abdomen, perineum, and vagina were prepared, painted, and draped after urinary catheterization. A $1.5-\mathrm{cm}$ vertical incision (modified Hassen's technique) was created in the skin of the umbilicus using a scalpel. After insertion of a wound retractor to the peritoneal cavity, the Lapsingle (Sejong Medical, Paju, Korea) was fixed to the outer ring of the wound retractor. The abdomen was insufflated with approximately 2 liters of $\mathrm{CO}_{2}$ gas, and the intra-abdominal pressure was maintained at $12 \mathrm{mmHg}$ throughout the surgery. In cases of robot-assisted LESS, the same technique was used, employing the DaVinci Xi System (Intuitive Surgical, Sunnyvale, (A, USA), except the size of the trocar-site vertical incision was approximately $2 \mathrm{~cm}$. At the end of the procedure after removal of the wound retractor, $10 \mathrm{~mL}$ of $0.25 \%$ bupivacaine hydrochloride was injected using a 22-gauge needle to the $1.5-\mathrm{cm}$ umbilical incision site to cover all preperitoneal layers in the injection group. Conversely, the umbilical trocar insertion site was closed without bupivacaine injection in the control group. IV-PCA was applied to all patients as a routine coverage before the end of surgery by the anesthesiologist following the protocol. The operation time was defined as the time from umbilical skin incision to closure.

\section{Postoperative pain score}

For pain score analysis, a $10-\mathrm{cm}$ VAS was used ( $0=$ no pain, $10=$ intractable and unbearable pain). A trained nurse prac- titioner blinded to bupivacaine usage gathered all the VAS scores. The pain scores were assessed at the time of the patients' return to the ward (2-3 hours after surgery), evening round of the surgery day (6-10 hours after surgery), morning round of the first postoperative day, and morning round of the third postoperative day.

\section{Statistical analysis}

The primary end point of this study was the difference in the VAS scores between the bupivacaine injection and noninjection groups. Assuming that a mean difference of 2 VAS scores with a standard deviation of 2.5 is clinically relevant, a sample size of 83 per group yielded a statistical power of $90 \%$ based on a 2-sided test with a 0.05 significance level. Considering the possible loss of patients during the followup period, 100 patients in each group were recruited [12]. For comparison of continuous variables, such as age and BMI, Student's $t$-test was used. For comparison of categorical variables, the $\chi^{2}$ test was used. The postoperative pain scores were compared between the 2 groups after adjusting for the confounding variables using a linear mixed model. SPSS version 23.0 (SPSS Inc., Chicago, IL, USA) was used, and $P$-values of $<0.05$ were considered statistically significant.

\section{Results}

Of the 223 gynecologic surgery cases in our institution, 23 were excluded. The remaining 200 LESS cases were alternatively

Table 1. Clinical characteristics

\begin{tabular}{lccc}
\hline Clinical characteristics & Bupivacaine $(\mathbf{n = 1 0 0 )}$ & No bupivacaine $(\mathbf{n}=\mathbf{1 0 0})$ & $\boldsymbol{P}$-value \\
\hline Age $(\mathrm{yr})$ & $49.2 \pm 10.8$ & $44.3 \pm 12.7$ & $0.004^{\mathrm{a})}$ \\
Parity & $1.7 \pm 0.9$ & $1.4 \pm 1.2$ & 0.062 \\
BMI $\left(\mathrm{kg} / \mathrm{m}^{2}\right)$ & $25.1 \pm 4.4$ & $23.7 \pm 3.6$ & $0.018^{\mathrm{a})}$ \\
Menopause & 31 & 19 & $0.045^{\mathrm{a})}$ \\
Associated comorbidities & 59 & 57 & 0.055 \\
ASA score & $1.7 \pm 0.8$ & $1.5 \pm 0.6$ & 0.091 \\
Previous abdominal surgery & $0.7 \pm 0.9$ & $0.6 \pm 0.9$ & 0.380 \\
Abdominal pain before surgery (VAS score) & $0.6 \pm 1.4$ & $0.7 \pm 1.3$ & 0.786 \\
\hline
\end{tabular}

Values are presented as number or mean \pm standard deviation.

BMI, body mass index; Comorbidities, hypertension, diabetes mellitus, hepatitis, hypercholesterolemia, and rheumatic disorders; ASA, American Society of Anesthesiologist; VAS, visual analog scale.

a) $P<0.05$. 


\section{Obstetrics \& Gynecology Science}

Vol. 63, No. 3, 2020

Table 2. Surgical characteristics

\begin{tabular}{|c|c|c|c|c|}
\hline \multicolumn{2}{|l|}{ Surgical characteristics } & \multirow{2}{*}{$\frac{\text { Bupivacaine }}{6}$} & \multirow{2}{*}{$\begin{array}{c}\text { No bupivac aine } \\
18\end{array}$} & \multirow{2}{*}{$\begin{array}{r}\boldsymbol{P} \text {-value } \\
0.026^{\mathrm{a})}\end{array}$} \\
\hline Surgery performed & Robotic hysterectomy & & & \\
\hline & Laparoscopic hysterectomy & 62 & 40 & \\
\hline & Robotic adnexa surgery & 2 & 4 & \\
\hline & Laparoscopic adnexal surgery & 25 & 28 & \\
\hline & Robotic myomectomy & 3 & 8 & \\
\hline & Laparoscopic myomectomy & 0 & 1 & \\
\hline & Robotic TLH + colpopexy & 1 & 1 & \\
\hline & Laparoscopic TLH + colpopexy & 1 & 0 & \\
\hline \multirow[t]{6}{*}{ Pathologic diagnosis } & Leiomyoma or adenomyosis & 61 & 61 & 0.642 \\
\hline & Endometriosis & 3 & 1 & \\
\hline & Benign ovarian neoplasm & 24 & 31 & \\
\hline & Cervical dysplasia & 7 & 4 & \\
\hline & Cancer & 4 & 2 & \\
\hline & Other & 1 & 1 & \\
\hline \multirow[t]{7}{*}{ Blood loss (mL) } & Minimal & 26 & 27 & 0.880 \\
\hline & $<100$ & 50 & 49 & \\
\hline & $<200$ & 14 & 13 & \\
\hline & $<300$ & 4 & 4 & \\
\hline & $<400$ & 1 & 4 & \\
\hline & $<500$ & 2 & 1 & \\
\hline & $\geq 500$ & 3 & 2 & \\
\hline \multirow[t]{6}{*}{ Hemoglobin decrease } & $0-0.9$ & 8 & 5 & 0.211 \\
\hline & $1-1.9$ & 33 & 31 & \\
\hline & $2-2.9$ & 36 & 35 & \\
\hline & $3-3.9$ & 17 & 19 & \\
\hline & $4-4.9$ & 2 & 9 & \\
\hline & $\geq 5$ & 4 & 1 & \\
\hline \multirow[t]{2}{*}{ Need for additional trocar } & No additional trocar & 92 & 87 & $0.003^{\mathrm{a})}$ \\
\hline & Additional trocar & 8 & 13 & \\
\hline \multirow[t]{3}{*}{ Perioperative complication } & None & 99 & 96 & 0.359 \\
\hline & Bowel injury & 0 & 1 & \\
\hline & Postoperative infection & 1 & 3 & \\
\hline \multicolumn{2}{|l|}{ Operation time in minutes } & $99.40 \pm 59.50$ & $109.18 \pm 61.20$ & 0.254 \\
\hline \multicolumn{2}{|l|}{ Packed RBC transfusion } & $0.06 \pm 0.34$ & $0.03 \pm 0.22$ & 0.464 \\
\hline \multicolumn{2}{|c|}{ Postoperative analgesic (ampules) } & $1.80 \pm 1.56$ & $1.79 \pm 1.54$ & 0.964 \\
\hline \multicolumn{2}{|c|}{ Hospital stay after surgery (day) } & $3.55 \pm 0.99$ & $3.51 \pm 1.31$ & 0.807 \\
\hline \multicolumn{2}{|c|}{ Time of flatus (hours after surgery) } & $34.49 \pm 13.00$ & $32.95 \pm 13.00$ & 0.404 \\
\hline
\end{tabular}

Values are presented as number or mean \pm standard deviation.

$\mathrm{TLH}$, total laparoscopic hysterectomy; RBC, red blood cell.

a) $P<0.05$. 


\section{Obstetrics \& Gynecology Science}

Jong Wook Seo, et al. Pain management in laparoscopic surgery

assigned to either the bupivacaine injection group or the non-injection group (Fig. 1).

In the comparison of the clinical characteristics between the bupivacaine injection group and non-injection group, the mean age $(49.2 \pm 10.8$ vs. $44.3 \pm 12.7$ years), BMI ( $25.1 \pm 4.4$ vs. $23.7 \pm 3.6 \mathrm{~kg} / \mathrm{m}^{2}$ ), and menopausal status (31 vs. 19 patients) exhibited significant differences (Table 1).

The most commonly performed surgery was hysterectomy. Of the 126 hysterectomy cases, 102 were performed with LESS, and 24 were accomplished by robot-assisted LESS. The mean operation time was $99.4 \pm 59.5$ minutes in the bupivacaine injection group and $109.2 \pm 61.2$ minutes in the noninjection group. There was no difference in blood loss during surgery, perioperative complications, hospital stay after surgery, or time of flatus between the 2 groups (Table 2).

After adjusting for the confounding variables of patient age, BMI, parity, menopausal status, ASA score, comorbidities, type of surgery performed, and need for additional trocar insertion, we found no significant difference in the postoperative umbilical VAS pain scores between the bupivacaine injection group and non-injection group. The comparison of postoperative umbilical pain in the hysterectomy cases showed no significant difference (Table 3).

For a sub-analysis, the postoperative pain scores were compared between the LESS $(n=157)$ and robot-assisted LESS $(\mathrm{n}=43)$ cases. The results were adjusted for the confounding variables of patient age, BMI, parity, menopausal status, ASA score, comorbidities, type of surgery performed, need for additional trocar usage, and bupivacaine injection. The pain score at the time of return to the ward was higher in the robot-assisted LESS cases than in the LESS cases $(4.6 \pm 1.0$ vs. $4.0 \pm 1.1, P=0.013)$. At the evening round on the surgery day,

Table 3. Comparison of the visual analog scale (VAS) scores between the bupivacaine injection and non-injection groups

\begin{tabular}{|c|c|c|c|}
\hline Characteristics & Bupivacaine & No bupivacaine & $P$-value \\
\hline Comparison of VAS in all laparoendoscopic procedures & $\mathrm{n}=100$ & $n=100$ & \\
\hline Ward return postoperative $2-3 \mathrm{hr}$ & $3.99 \pm 1.07$ & $4.27 \pm 1.25$ & 0.263 \\
\hline Evening round postoperative $6-10 \mathrm{hr}$ & $3.37 \pm 1.06$ & $3.54 \pm 1.06$ & 0.801 \\
\hline POD\#1 morning round & $2.64 \pm 0.87$ & $2.89 \pm 1.06$ & 0.336 \\
\hline POD\#3 morning round & $1.75 \pm 0.54$ & $1.81 \pm 0.72$ & 0.627 \\
\hline Comparison of VAS in hysterectomy procedures & $n=68$ & $n=58$ & \\
\hline Ward return postoperative $2-3 \mathrm{hr}$ & $3.99 \pm 0.98$ & $4.28 \pm 1.24$ & 0.359 \\
\hline Evening round postoperative $6-10 \mathrm{hr}$ & $3.28 \pm 0.91$ & $3.38 \pm 0.93$ & 0.823 \\
\hline POD\#1 morning round & $2.65 \pm 0.93$ & $2.83 \pm 1.06$ & 0.812 \\
\hline POD\#3 morning round & $1.74 \pm 0.54$ & $1.86 \pm 0.80$ & 0.594 \\
\hline
\end{tabular}

The VAS score is presented as mean \pm standard deviation. Adjusted for age, body mass index, parity, menopausal status, American Society of Anesthesiology score, comorbidities, type of surgery, and need for additional trocar usage.

POD, postoperative day.

Table 4. Comparison of the visual analog scale (VAS) score between the laparoendoscopic single-site surgery (LESS) and robot-assisted LESS cases

\begin{tabular}{|c|c|c|c|}
\hline Comparison of VAS between LESS and robot-assisted LESS & LESS $(n=157)$ & Robot-assisted LESS $(n=43)$ & P-value \\
\hline Ward return postoperative $2-3 \mathrm{hr}$ & $3.99 \pm 1.16$ & $4.63 \pm 1.01$ & $0.013^{\mathrm{a})}$ \\
\hline Evening round postoperative 6-10 hr & $3.33 \pm 1.04$ & $3.91 \pm 1.02$ & $0.021^{\mathrm{a})}$ \\
\hline POD\#1 morning round & $2.70 \pm 0.91$ & $3.00 \pm 1.18$ & 0.787 \\
\hline POD\#3 morning round & $1.73 \pm 0.59$ & $1.96 \pm 0.76$ & 0.930 \\
\hline
\end{tabular}

The VAS score is presented as mean \pm standard deviation. Adjusted for age, body mass index, parity, menopausal status, American Society of Anesthesiology score, comorbidities, type of surgery, need for additional trocar usage, and bupivacaine usage.

POD, postoperative day.

${ }^{\text {a) }} P<0.05$. 


\title{
Obstetrics \& Gynecology Science
}

\author{
Vol. 63, No. 3, 2020
}

the difference in the pain scores between the robot-assisted LESS and LESS cases persisted ( $3.9 \pm 1.0$ vs. $3.3 \pm 1.0, P=0.021$ ) (Table 4).

\section{Discussion}

Surgical techniques in the field of gynecologic surgery have shifted from the conventional open transabdominal approach to LESS, where laparoendoscopic procedures are performed through a single umbilical incision. LESS has been shown to yield numerous benefits, such as decreased postoperative pain, shorter hospital stay, and faster recovery to normal daily activities [14-19]. As the incision is created in the umbilicus, the surgical scar is concealed, providing aesthetic superiority. Numerous studies have analyzed the safety and effectiveness of LESS, and this technique is used not only for benign adnexal surgeries but also for hysterectomies and cancer surgeries $[20,21]$. Most of the gynecologic surgeries performed in the Department of Obstetrics and Gynecology, National Health Insurance Service Ilsan Hospital are conducted with a standardized procedure of LESS or robotassisted LESS (DaVinci Xi System). As a part of the effort to optimize postoperative patient care in LESS, we launched this prospective, randomized study with the hypothesis that patients receiving port-site local anesthetic injection would experience less postoperative umbilical pain than controls. As all patients received IV-PCA for general postoperative pain alleviation, we sought to analyze the additional role of trocarsite bupivacaine injection in specifically moderating incisional umbilical pain.

Previous reports on the effectiveness of trocar-site local anesthetic injection in LESS included only adnexal procedures lasting less than 1 hour, and the role of port-site bupivacaine injection in more complex surgeries with longer operation times accompanied by comparatively higher postoperative VAS scores remains uncertain [13]. In this prospective study, we sought to include all consecutively performed gynecologic laparoendoscopic surgeries, including hysterectomies, myomectomies, cancer surgeries, and robot-assisted procedures, to analyze the feasibility of trocar-site bupivacaine injection as a routine postoperative procedure in addition to the application of routine IV-PCA. During the 4-month study period, the most commonly performed procedure was hysterectomy, not adnexal surgery. The operation time was longer in the present study than in studies based on adnexal surgeries (Tables 1 and 2). Instead of being conducted by a single surgeon, LESS was performed by 3 board-certified gynecologists, allowing for generalizability of the technique. Although this study was not double-blinded, the postoperative pain scores were gathered by a nurse practitioner who was blinded to the usage of bupivacaine to avoid observerexpectancy bias. As the surgeons were not involved in the postoperative pain evaluation, they were not blinded to this process.

Bupivacaine hydrochloride is a relatively safe local anesthetic; however, cardiotoxic symptoms, such as palpitations, and neurotoxic symptoms, including circumoral numbness or muscle fasciculations, may occur [22]. The patients included in this study had no history of bupivacaine allergy, and no drop out was noted owing to bupivacaine complications. Compared to bupivacaine hydrochloride, liposomal bupivacaine composed of multi-vesicular liposomes has yielded increased plasma concentrations over a longer period [23-25]. Further studies exploring various postoperative pain modulators, such as liposomal bupivacaine, might shed new light on postoperative pain management in LESS.

This randomized, prospective study involving 200 LESS procedures revealed no postoperative umbilical pain alleviation effect after trocar-site bupivacaine injection. The analysis including only the hysterectomy cases showed no additive umbilical pain alleviation effect in the bupivacaine injection group (Table 3). For the sub-group analysis, we compared the postoperative pain scores between the LESS $(n=157)$ and robot-assisted LESS ( $n=43$ ) cases. The postoperative umbilical pain scores at return to the ward and at the evening round on the surgery day were significantly higher in the robotassisted LESS cases than in the LESS cases (Table 4).

In conclusion, trocar-site bupivacaine injection in patients who undergo LESS does not have an additive role in postoperative umbilical pain management. With its simplicity and economic feasibility, the routine usage of postoperative trocar-site bupivacaine injection should be reconsidered as a means of reducing postoperative incisional pain.

\section{Conflict of interest}

No potential conflict of interest relevant to this article was reported. 


\section{Obstetrics \& Gynecology Science}

Jong Wook Seo, et al. Pain management in laparoscopic surgery

\section{Ethical approval}

This study was approved by the National Health Insurance Service Ilsan Hospital Institutional Review Board (NHIS IRB 2018-04-017).

\section{Patient consent}

Detailed explanation about the planned procedure was given to the patient by the surgeon and written consent form was collected from all patients.

\section{References}

1. Khavanin N, Mlodinow A, Milad MP, Bilimoria KY, Kim JY. Comparison of perioperative outcomes in outpatient and inpatient laparoscopic hysterectomy. J Minim Invasive Gynecol 2013;20:604-10.

2. Wattiez A, Cohen SB, Selvaggi L. Laparoscopic hysterectomy. Curr Opin Obstet Gynecol 2002;14:417-22.

3. Pitter MC, Simmonds C, Seshadri-Kreaden U, Hubert HB. The impact of different surgical modalities for hysterectomy on satisfaction and patient reported outcomes. Interact J Med Res 2014;3:e11.

4. Medeiros LR, Rosa DD, Bozzetti MC, Fachel JM, Furness S, Garry R, et al. Laparoscopy versus laparotomy for benign ovarian tumour. Cochrane Database Syst Rev 2009:CD004751.

5. Marks JL, Ata B, Tulandi T. Systematic review and metaanalysis of intraperitoneal instillation of local anesthetics for reduction of pain after gynecologic laparoscopy. J Minim Invasive Gynecol 2012;19:545-53.

6. Parsanezhad ME, Lahsaee $M$, Alborzi S, Vafaei $H$, Schmidt EH. Comparative, double-blind, randomized, placebo-controlled trial of intraperitoneal of bupivacaine and lidocaine for pain control after diagnostic laparoscopy. J Am Assoc Gynecol Laparosc 2003;10:311-5.

7. Saleh A, Fox G, Felemban A, Guerra C, Tulandi T. Effects of local bupivacaine instillation on pain after laparoscopy. J Am Assoc Gynecol Laparosc 2001;8:203-6.

8. Lam KW, Pun TC, Ng EH, Wong KS. Efficacy of preemptive analgesia for wound pain after laparoscopic operations in infertile women: a randomised, double-blind and placebo control study. BJOG 2004;111:340-4.

9. Roy KK, Subbaiah M, Naha M, Kumar S, Sharma JB, Jahagirdar N. Intraperitoneal bupivacaine for pain relief after minilaparoscopy in patients with infertility. Arch Gynecol Obstet 2014;289:337-40.

10. Ghezzi F, Cromi A, Bergamini V, Raffaelli R, Crotti S, Segredini $R$, et al. Preemptive port site local anesthesia in gynecologic laparoscopy: a randomized, controlled trial. J Minim Invasive Gynecol 2005;12:210-5.

11. Savaris RF, Chicar LL, Cristovam RS, Moraes GS, Miguel OA. Does bupivacaine in laparoscopic ports reduce postsurgery pain in tubal ligation by electrocoagulation? A randomized controlled trial. Contraception 2010;81:542-6.

12. Tam T, Harkins G, Wegrzyniak L, Ehrgood S, Kunselman A, Davies M. Infiltration of bupivacaine local anesthetic to trocar insertion sites after laparoscopy: a randomized, double-blind, stratified, and controlled trial. J Minim Invasive Gynecol 2014;21:1015-21.

13. Kim JE, Shim SH, Dong M, Lee H, Hwang HS, Kwon $H S$, et al. Port site infiltration of local anesthetic after laparoendoscopic single site surgery for benign adnexal disease. Obstet Gynecol Sci 2017;60:455-61.

14. Lee YY, Kim TJ, Kim CJ, Park HS, Choi CH, Lee JW, et al. Single port access laparoscopic adnexal surgery versus conventional laparoscopic adnexal surgery: a comparison of peri-operative outcomes. Eur J Obstet Gynecol Reprod Biol 2010;151:181-4.

15. Hoyer-Sørensen C, Vistad I, Ballard K. Is single-port laparoscopy for benign adnexal disease less painful than conventional laparoscopy? A single-center randomized controlled trial. Fertil Steril 2012;98:973-9.

16. Teoh AY, Chiu PW, Wong TC, Poon MC, Wong SK, Leong $H T$, et al. A double-blinded randomized controlled trial of laparoendoscopic single-site access versus conventional 3-port appendectomy. Ann Surg 2012;256:909-14.

17. Sangnier E, Lallemant M, Gnofam M, Bednarczyk L, Mereb E, Graesslin O, et al. Single port laparoscopy (SPL): retrospective study evaluating postoperative pain in comparison with conventional laparoscopy (CL). J Gynecol Obstet Hum Reprod 2018;47:365-9.

18. Fagotti A, Bottoni C, Vizzielli G, Gueli Alletti S, Scambia $G$, Marana $E$, et al. Postoperative pain after conventional laparoscopy and laparoendoscopic single site surgery 


\section{Obstetrics \& Gynecology Science}

Vol. 63, No. 3, 2020

(LESS) for benign adnexal disease: a randomized trial. Fertil Steril 2011;96:255-9.e2.

19. Mencaglia L, Mereu L, Carri G, Arena I, Khalifa H, Tateo S, et al. Single port entry - Are there any advantages? Best Pract Res Clin Obstet Gynaecol 2013;27:441-55.

20. Fader AN, Rojas-Espaillat L, Ibeanu O, Grumbine FC, Escobar PF. Laparoendoscopic single-site surgery (LESS) in gynecology: a multi-institutional evaluation. Am J Obstet Gynecol 2010;203:501.e1-6.

21. Figurelli J, Bresson L, Narducci F, Katdare N, Coulon P, Fournier $C$, et al. Single-port access laparoscopic surgery in gynecologic oncology: outcomes and feasibility. Int J Gynecol Cancer 2014;24:1126-32.

22. Balasanmugam C, Henriquez Felipe C, Rodriguez D, Kulbak G. Bradycardia, hypotension, and cardiac ar- rest: a complication of local anesthetics. Cureus 2019;11:e4033.

23. Beiranvand S, Moradkhani MR. Bupivacaine versus liposomal bupivacaine for pain control. Drug Res (Stuttg) 2018;68:365-9.

24. Yan Z, Chen Z, Ma C. Liposomal bupivacaine versus interscalene nerve block for pain control after shoulder arthroplasty: a meta-analysis. Medicine (Baltimore) 2017; 96:e7226.

25. Sun H, Li S, Wang K, Zhou J, Wu G, Fang S, et al. Do liposomal bupivacaine infiltration and interscalene nerve block provide similar pain relief after total shoulder arthroplasty: a systematic review and meta-analysis. J Pain Res 2018;11:1889-900. 